EPJ Web of Conferences 73, 05003 (2014)

DOI: $10.1051 /$ epjconf/20147305003

(C) Owned by the authors, published by EDP Sciences, 2014

\title{
A search for the deeply bound kaonic nuclear state at J-PARC
}

\author{
M. Tokuda ${ }^{\mathrm{a}}$ for the J-PARC E15 collaboration ${ }^{\mathrm{b}}$ \\ Department of Physics, Tokyo Institute of Technology, Tokyo 152-8551, Japan
}

\begin{abstract}
The J-PARC E15 experiment aims to search for the simplest kaonic nuclear bound state, $K^{-} p p$, by the in-flight ${ }^{3} \mathrm{He}\left(K^{-}, n\right)$ reaction. In May 2013 , the first physics data were taken at the K1.8BR beam-line in the J-PARC hadron hall. In this report, results of the semi-inclusive ${ }^{3} \mathrm{He}\left(K^{-}, n\right)$ and the exclusive ${ }^{3} \mathrm{He}\left(K^{-}, \Lambda p n\right)$ channels are discussed.
\end{abstract}

\section{Introduction}

The $\bar{K} \mathrm{~N}$ interaction has been shown to be strongly attractive by extensive measurements of the kaonic hydrogen atom [1] and low-energy $\bar{K} \mathrm{~N}$ scattering [2]. As a key to deepen the understanding of the strongly attractive $\mathrm{I}=0 \bar{K} \mathrm{~N}$ interaction, existence of deeply-bound $\bar{K}$-nuclear states has been widely discussed in the recent years. In particular, the simplest $\bar{K}$-nuclear bound system, $K^{-} p p$, has been theoretically supported. However, available experimental information is limited and the reported $K^{-} p p$ masses and widths are scattered and conflict with each other [3]. In order to clarify this controversial issue, we study the $K^{-} p p$ system via the ${ }^{3} \mathrm{He}\left(K^{-}, n\right)$ reaction.

The E15 experiment aims to search for the simplest $\bar{K}$-nuclear bound state [4], $K^{-} p p$, by reconstructing the complete kinematics of the reaction channels to discriminate all background processes. An exclusive measurement is performed with the in-flight ${ }^{3} \mathrm{He}\left(K^{-}, n\right)$ reaction at $p_{k}=1.0$ $\mathrm{GeV} / \mathrm{c}$. This reaction permits performing a missing-mass study using the produced neutron, and invariant-mass spectroscopy via the decay chain $K^{-} p p \rightarrow \Lambda p \rightarrow p \pi^{-} p$, simultaneously.

\section{The E15 spectrometer}

The E15 experiment is performed at the K1.8BR beam-line in the Hadron Experimental Hall at the J-PARC (the Japan Proton Accelerator Research Complex). The layout of K1.8BR and the E15

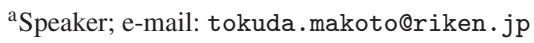

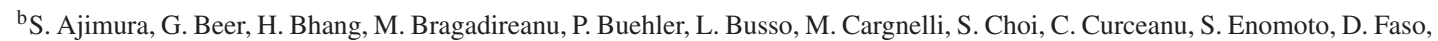
H. Fujioka, Y. Fujiwara, T. Fukuda, C. Guaraldo, T. Hashimoto, R.S. Hayano, T. Hiraiwa, M. Iio, M. Iliescu, K. Inoue, Y. Ishiguro, T. Ishikawa, S. Ishimoto, T. Ishiwatari, K. Itahashi, M. Iwai, M. Iwasaki, Y. Kato, S. Kawasaki, P. Kienle, H. Kou, Y. Ma, J. Marton, Y. Matsuda, Y. Mizoi, O. Morra, T. Nagae, H. Noumi, H. Ohnishi, S. Okada, H. Outa, K. Piscicchia, M. Poli Lener, A. Romero Vidal, Y. Sada, A. Sakaguchi, F. Sakuma, M. Sato, A. Scordo, M. Sekimoto, H. Shi, D. Sirghi, F. Sirghi, K. Suzuki, S. Suzuki, T. Suzuki, K. Tanida, H. Tatsuno, D. Tomono, A. Toyoda, K. Tsukada, O. Vazquez Doce, E. Widmann, B.K. Wuenschek, T. Yamaga, T. Yamazaki, H. Yim, Q. Zhang, and J. Zmeskal
}

This is an Open Access article distributed under the terms of the Creative Commons Attribution License 4.0, which permits unrestricted use, distribution, and reproduction in any medium, provided the original work is properly cited. 


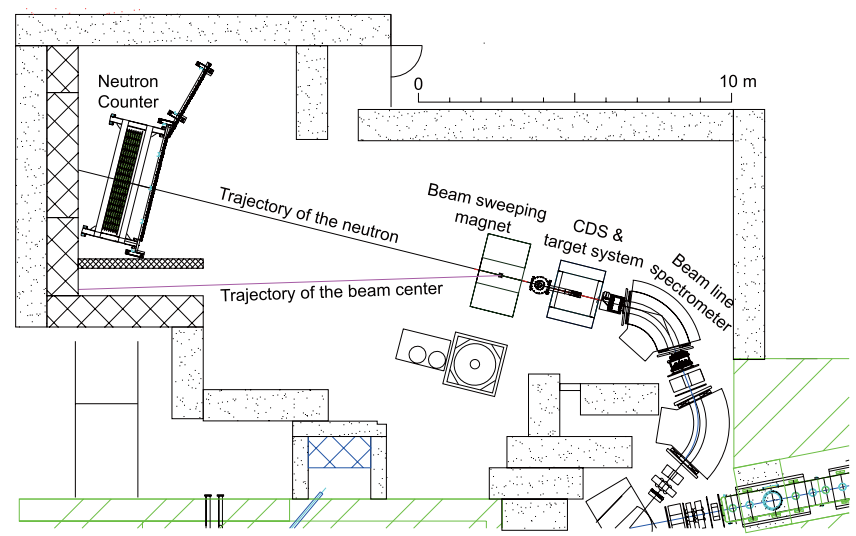

Figure 1. Schematic view of the K1.8BR spectrometer.

spectrometer are shown in Fig. 1. The experimental setup consists of three parts: a high precision beam line spectrometer, a cylindrical detector system (CDS) around the liquid ${ }^{3} \mathrm{He}$ target system, and a forward neutron TOF counter (NC) located about $15 \mathrm{~m}$ downstream from the center of the target. A typical event is shown in the following. The $1.0 \mathrm{GeV} / \mathrm{c}$ kaon beam ran through the beam line and was focused on the ${ }^{3} \mathrm{He}$ target. After the ${ }^{3} \mathrm{He}\left(K^{-}, n\right)$ reaction, particles produced from the $K^{-} p p$ were detected by CDS and the generated forward neutron was detected by using the NC.

The kaon in the beam was identified by using an Aerogel Cherenkov counter. The beam momentum was analyzed by the beam line spectrometer whose momentum resolution is $2.2 \mathrm{MeV} / \mathrm{c}$ at $1.0 \mathrm{GeV} / \mathrm{c}$. The CDS was placed surrounding the target in order to identify the $\Lambda p$ final state from the $K^{-} p p$ state. The CDS consists of a solenoid magnet, a Cylindrical Drift Chamber (CDC), and a Cylindrical Detector Hodoscope $(\mathrm{CDH})$ and has a solid angle coverage of $59 \%$ of $4 \pi$. The details of the CDS system can be found in a separate paper [5]. Tracking information of charged particles was obtained from the CDC which operated in a solenoidal magnetic field of $0.7 \mathrm{~T}$. Particle identification was obtained using timeof-flight (TOF). Figure 2 shows the distribution of the momentum versus $1 / \beta$ reconstructed in the CDS, where $\pi^{ \pm}, K^{-}, p$ and $d$ were separately identified. The invariant mass of $\mathrm{p}$ and $\pi^{-}$was reconstructed as shown in Fig. 3. A clear peak of $\Lambda \rightarrow p \pi^{-}$decay was observed. The NC, placed $15 \mathrm{~m}$ downstream from the center of the target at 0 degrees with respect to the beam direction, detected forward neutrons generated by the in-flight $\left(K^{-}, n\right)$ reaction. A $1 / \beta$ spectrum of neutral particles detected by the $\mathrm{NC}$ is shown in Fig. 4. Neutrons are clearly separated from gamma rays. A signal to noise ratio is found to be about 100 in the vicinity of the neutron peak. The TOF resolution was measured to be 150 ps $(\sigma)$ at the gamma peak. A resolution of the $K^{-} p p$ state in the missing mass of the ${ }^{3} \mathrm{He}\left(K^{-}, n\right)$ reaction is estimated to be $9 \mathrm{MeV} / \mathrm{c}^{2}$, which satisfies the experimental requirement of less than $10 \mathrm{MeV} / \mathrm{c}^{2}(\sigma)$.

\section{Preliminary results of the first physics run}

The first physics-run of the E15 experiment was carried out in May 2013 . By irradiating $5 \times 10^{9}$ kaons on the helium-3 target, $3 \times 10^{5}$ forward-neutrons were successfully recorded. Accumulated data corresponds to about $1 \%$ of statistics requested in the original proposal. Fig. 5 shows the missing mass of the ${ }^{3} \mathrm{He}\left(K^{-}, n\right)$ reaction measured by the NC. One or more charged tracks are required in the CDS to reconstruct the reaction vertex. In the spectrum, a clear peak from the quasi-elastic $K^{-} n \rightarrow K^{-} n$ and the charge exchange $K^{-} p \rightarrow K_{s}^{0} n$ reactions on ${ }^{3} \mathrm{He}$ is observed. The spectrum with $K_{s}^{0}$ reconstructed in the CDS is overlaid in the figure, which is distributed above the $K^{-} p p$ threshold $\left(2.37 \mathrm{GeV} / c^{2}\right)$. The spectrum shape of the quasi-elastic / charge exchange reaction is well reproduced by a Monte 


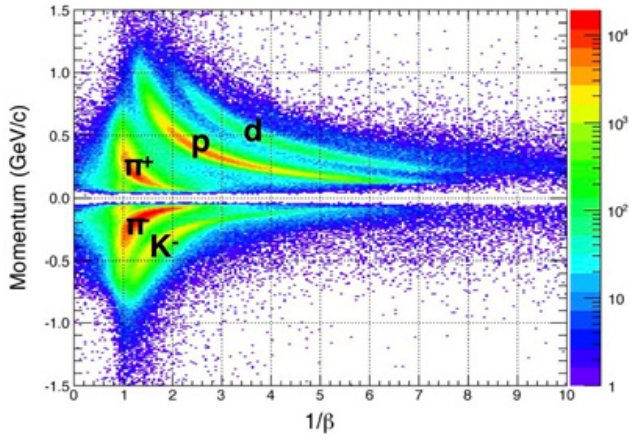

Figure 2. Distribution of the momentum versus $1 / \beta$ obtained by the CDS.

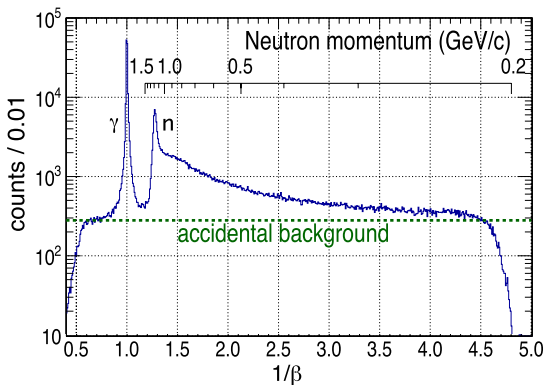

Figure 4. $1 / \beta$ spectrum of neutral particles detected by the NC. The dotted line shows an accidental background evaluated from the left shoulder of the $\gamma$ peak at $1 / \beta=1$.

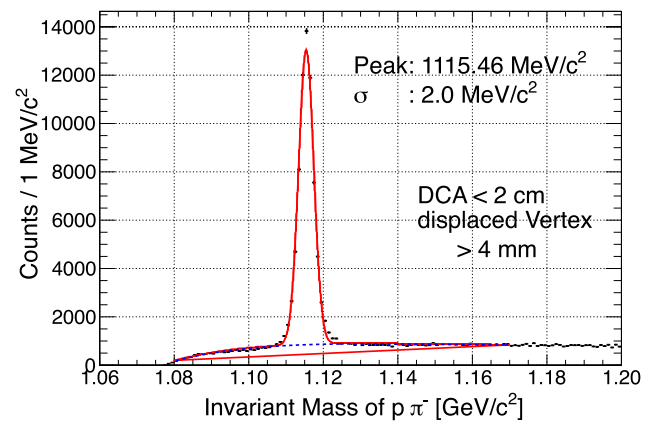

Figure 3. Invariant mass spectrum of $\mathrm{p} \pi^{-}$. The spectrum is fitted with a Gaussian and a background curve.

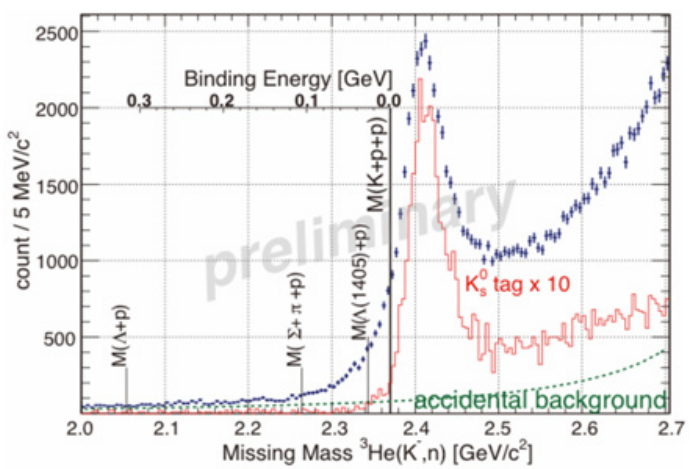

Figure 5. Missing mass of the ${ }^{3} \mathrm{He}\left(K^{-}, n\right)$ reaction at forward angle. One or more charged tracks are required in the CDS to reconstruct the reaction vertex. A spectrum with $K_{s}^{0}$ reconstructed in the CDS is overlaid with a scale factor of 10 .

Carlo simulation. The excess below the $K^{-} p p$ threshold in the inclusive ${ }^{3} \mathrm{He}\left(K^{-}, n\right)$ spectrum is poorly explained by the detector responses. It may indicate presence of a bound state $K^{-} p p$, but, to elucidate this, we need more statistics.

The missing mass of ${ }^{3} \mathrm{He}\left(K^{-}, \Lambda p\right)$ reaction is shown in Fig. 6. A peak at the neutron mass is seen in the spectrum. By gating the missing neutron mass region, we select the events of the $\Lambda p n$ final state. A Dalitzs plot of $\Lambda p n$ is shown in Fig. 7. We found that the selected $\Lambda p n$ events are widely distributed over the kinematically allowed phase space. Most of the events have no correlation among three final state particles. If the neutron detection by the NC (namely, at forward direction) is required, only a few events are left. It is necessary for $K^{-} p p$ to carry out experiments with higher statistics. Further analysis is in progress to understand the observed spectrum.

\section{Summary}

The J-PARC E15 experiment was performed to search for the simplest kaonic nuclear bound state, $K^{-} p p$, by the in-flight ${ }^{3} \mathrm{He}\left(K^{-}, n\right)$ reaction at $1 \mathrm{GeV} / \mathrm{c}$. The first physics data-taking was in May 2013. 


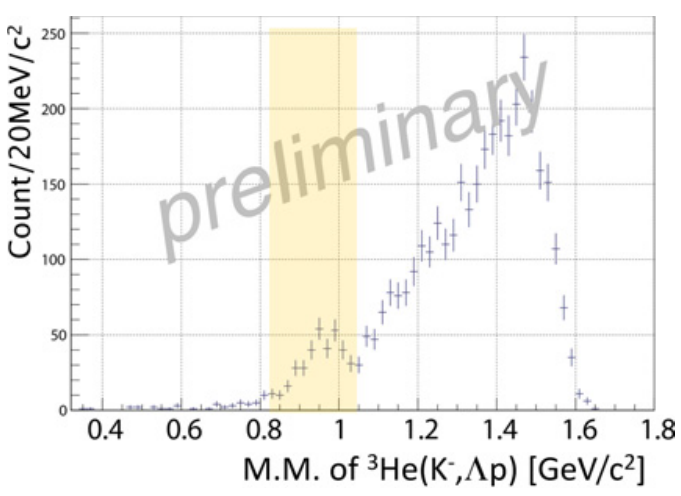

Figure 6. The missing mass of ${ }^{3} \mathrm{He}\left(K^{-}, \Lambda p\right)$ reaction. Hatched area represents the selecting region of missing neutrons $\left(0.85<M . M .<1.05 G e V / c^{2}\right)$.

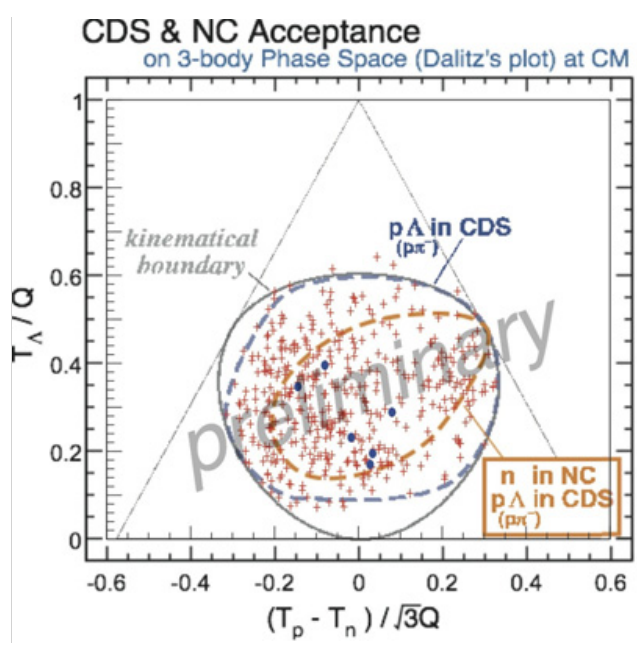

Figure 7. Dalitz plot of $\Lambda p n$ in the exclusive $\Lambda p n$ events (red point) whose neutron is selected by ${ }^{3} \mathrm{He}\left(K^{-}, \Lambda p\right)$ missing mass.If the neutron detection by the $\mathrm{NC}$ is required, only a few events are left (blue point). The area surrounded by the blue dotted line is the expected sensitive area of the CDS, and also the area surrounded by the yellow line required the signal of NC.

$5 \times 10^{9}$ kaons were incident on the ${ }^{3} \mathrm{He}$ target, and $3 \times 10^{5}$ neutrons were recorded. The semi-inclusive ${ }^{3} \mathrm{He}\left(K^{-}, n\right)$ spectrum shows clear peak structure composed of the quasi-elsastic $K^{-} n \rightarrow K^{-} n$ and the charge exchange $K^{-} p \rightarrow K_{s}^{0} n$ reactions as expected. The exclusive ${ }^{3} \mathrm{He}\left(K^{-}, \Lambda p n\right)$ analysis indicates 3-nucleon absorption processes are dominant. Further analyses of the semi-inclusive ${ }^{3} \mathrm{He}\left(K^{-}, n\right)$ and the exclusive ${ }^{3} \mathrm{He}\left(K^{-}, \Lambda p n\right)$ channels are in progress.

\section{References}

[1] M. Iwasaki, et al., Phys. Rev. Lett. 78, 3067 (1997)

G. Beer, et al., Phys. Rev. Lett. 94, 212302 (2005)

M. Bazzi, et al., Phys. Lett. B 704, 113 (2011)

[2] A.D. Martin, Nucl. Phys. B 179, 33 (1981)

[3] M. Agnello, et al., Phys. Rev. Lett. 94, 212303 (2005)

T. Yamazaki, et al., Phys. Rev. Lett. 104, 132502 (2010)

L. Fabbietti, et al., Nucl. Phys. A 914, 60 (2013)

A.O. Tokiyasu, et al., Phys. Lett. B 728C, 616-621 (2014)

[4] J-PARC E15 proposal : http://j-parc.jp/NuclPart/pac_0606/pdf/p15-Iwasaki.pdf

[5] K. Agari et al., Prog. Theor. Exp. Phys., 02B011 (2012) 